\title{
Effectiveness of GNSS Disposal Strategies
}

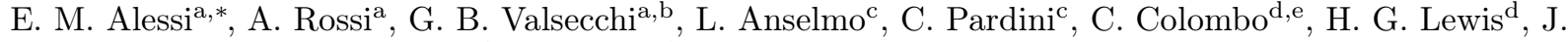

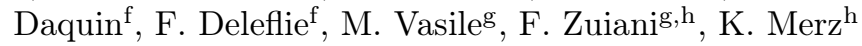 \\ ${ }^{a}$ IFAC-CNR, via Madonna del Piano 10, 50019 Sesto Fiorentino (FI), Italy \\ ${ }^{b}$ IAPS-INAF, via Fosso del Cavaliere 100, 00133 Roma, Italy \\ ${ }^{c}$ ISTI-CNR, via G. Moruzzi 1, 56124 Pisa, Italy \\ ${ }^{d}$ University of Southampton, Faculty of Engineering and the Environment, University Road, Southampton SO17 $1 B J$, UK \\ ${ }^{e}$ Politecnico di Milano, Aerospace Science and Technology Department, via La Masa 34, 20156 Milano, Italy

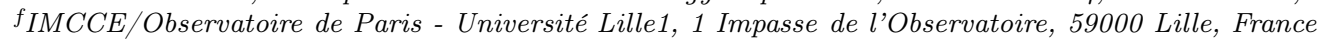 \\ ${ }^{g}$ University of Strathclyde, Mechanical \& Aerospace Engineering, James Weir Building, 75 Montrose Street, G11XJ \\ Glasgow, UK \\ ${ }^{h}$ ESA-ESOC, Space Debris Office, Robert-Bosch-Straße 5, 64293 Darmstadt, Germany
}

\begin{abstract}
The management of the Global Navigation Satellite Systems (GNSS) and of the Medium Earth Orbit (MEO) region as a whole is a subject that cannot be deferred, due to the growing exploitation and launch rate in that orbital regime. The advent of the European Galileo and the Chinese Beidou constellations significantly added complexity to the system and call for an adequate global view on the four constellations presently in operation. The operation procedures, including maintenance and disposal practices, of the constellations currently deployed were analyzed in order to asses a proper reference simulation scenario. The complex dynamics of the MEO region with all the geopotential and lunisolar resonances was studied to better identify the proper end-of-life orbit for every proposed strategy, taking into account and, whenever possible, exploiting the orbital dynamics in this peculiar region of space. The possibility to exploit low thrust propulsion or non gravitational perturbations with passive de-orbiting devices (and a combination of the two) was analyzed, in view of possible applications in the design of the future generations of the constellations satellites. Several upgrades in the long-term evolution software SDM and DAMAGE were undertaken to properly handle the constellation simulations in every aspect from constellation maintenance to orbital dynamics. A thorough approach considering the full time evolving covariance matrix associated to every object was implemented in SDM to compute the collision risk and associated maneuver rate for the constellation satellites. Once the software upgrades will be completed, the effectiveness of the different disposal strategies will be analyzed in terms of residual collision risk and avoidance maneuvers rate. This work was performed under the ESA/GSP Contract No. 4000107201/12/F/MOS.
\end{abstract}

Keywords: Space debris, orbital dynamics, Global Navigation Satellite Systems

\footnotetext{
* Corresponding author

Email addresses: em.alessi@ifac.cnr.it (E. M. Alessi), a.rossi@ifac.cnr.it (A. Rossi), giovanni@iaps.inaf.it (G. B. Valsecchi), luciano.anselmo@isti.cnr.it (L. Anselmo), carmen.pardini@isti.cnr.it (C. Pardini), c.colombo@soton.ac.uk (C. Colombo), hglewis@soton.ac.uk (H. G. Lewis), Jerome.Daquin@imcce.fr (J. Daquin), Florent.Deleflie@imcce.fr (F. Deleflie), massimiliano.vasile@strath.ac.uk (M. Vasile), federico.zuiani@strath.ac.uk (F. Zuiani), klaus.merz@esa.int (K. Merz)
}

Preprint submitted to Acta Astronautica

\section{Introduction}

A Global Navigation Satellite System (GNSS) consists of a spacecraft constellation able to provide autonomous geo-spatial positioning (and relative velocity), plus accurate timing, with global coverage. The current operational systems (GPS and GLONASS) are placed in Medium Earth Orbit (MEO) and are complemented by Satellite Based Augmentation Systems (SBAS), generally using spacecraft in geostationary orbits (GEO), and by Ground Based Augmentation Systems (GBAS). 
The new generation European navigation system (Galileo) and the MEO segment of the Chinese Beidou (Compass) system, are being deployed in MEO too. Therefore, due to the exploitation and launch rate in this orbital regime, the management of the GNSS, and of the MEO region as a whole, is a subject that cannot be deferred. A new global view on the four constellations presently in operation is in order. For this purpose an analysis of the effectiveness of the disposal practices in the MEO regime is ongoing under the ESA/GSP Contract No. 4000107201/12/F/MOS. The preliminary studies performed in this framework are presented in this paper.

\section{Navigation Satellite Systems in MEO}

A short description of the current and future MEO GNSS constellations, along with their operational and disposal practices, follows.

\section{1. $G P S$}

The American Global Positioning System (NAVSTAR-GPS) consists of a minimum of 24 operational satellites in near circular orbits 20182 $\mathrm{km}$ above the Earth. The constellation includes 6 orbital planes separated by $60^{\circ}$ at the equator and the satellites are spaced in orbit so that at any time a minimum of 4 satellites are in view to users anywhere in the world. Initially the orbit inclination was defined to be $63^{\circ}$ (Block I), but early in the program it was changed to $55^{\circ}$ to allow Space Shuttle launches. As of 8 July 2013, 61 GPS spacecraft have been successfully launched, from Block I and II to Block IIF models, and 31 spacecraft are currently operational, with a minimum of 5 and a maximum of 6 spacecraft per orbital plane. In addition, 3-4 old decommissioned satellites ("residuals") can be reactivated if needed. Since 1978, an average of 7 satellites every 4 years have been placed into orbit. The system became fully operational in 1994 and a new upgraded spacecraft model, Block III, should debut in 2014. The operational GPS satellites have revolution periods of half a sidereal day (i.e. $\simeq 718$ minutes), so they complete two orbital revolutions during one Earth rotation. For this reason they are in a "deep" (2:1) resonance with the Earth's rotation, dominated by the terms $J_{32}$ and $J_{44}$ of the geopotential [1]. The accumulated perturbation effect significantly depends on the initial longitude of the sub-satellite track and this means that satellites placed in the same orbital plane, but broadly spaced in the argument of latitude, are subjected to quite different perturbation effects, requiring one orbital maneuver per year, per satellite, in order to maintain the proper separation among them [1]. It should also be mentioned that the current nominal inclination of the GPS constellation is close to the "critical" value of $54.74^{\circ}$, i.e. over one revolution period the mean motion of a GPS spacecraft in the potential of an oblate Earth is the same, on average, as in the potential of a spherical Earth [1].

\subsection{GLONASS}

The Russian Globalnaya Navigatsionnaya Sputnikovaya Sistema or Global Navigation Satellite System (GLONASS) consists of a minimum of 24 operational satellites, plus 3 on-orbit spares, in near circular orbits $19130 \mathrm{~km}$ above the Earth. The constellation includes 3 orbital planes equally separated by $120^{\circ}$ at the equator and 8 satellites are evenly spaced in argument of latitude in each plane. The orbit inclination is around $65^{\circ}$. As of 8 July 2013, 125 GLONASS spacecraft have been successfully launched and 30 are currently active: 24 operational (Uragan-M), 3 spares (Uragan-M), 2 in maintenance (Uragan-M) and 1 in flight test phase (Uragan-K1). Since 1982, an average of 4 satellites per year have been placed into orbit. After the first deployment in 1995, the full constellation of 24 operational spacecraft was restored only in October 2011. Three spacecraft models have been launched so far: Uragan (1982), with a design lifetime of 4 years, Uragan-M (2003), with a design lifetime of 7 years, and Uragan-K1 (2011), with a design lifetime of 10 years. New improved versions will follow in the second half of the decade, with a design lifetime of 12 years: Uragan-K2 and Uragan-KM. The operational GLONASS satellites have revolution periods of about 676 minutes, therefore they complete 17 orbital revolutions during 8 Earth rotations. For this reason they are in a "shallow" (17:8) resonance with the Earth's rotation.

\subsection{Galileo}

The new European global navigation satellite system Galileo will consist of 30 operational satellites, including 6 spares, in near circular orbits $23222 \mathrm{~km}$ above the Earth. The Walker "delta" pattern constellation will include 3 orbital planes equally separated by $120^{\circ}$ at the equator and 8 operational 
satellites plus 2 spares in each plane. The selected orbit inclination is $56^{\circ}$. Due to station keeping, the right ascension of the ascending node of the satellites in each plane will be kept within $\pm 1^{\circ}$ with respect to the reference. The inclination will be maintained within $\pm 2^{\circ}$ of the reference. As of 8 July 2013, after 2 Galileo In-Orbit Validation Element (GIOVE) spacecraft, launched in 2005 and 2008 and now decommissioned, 4 In-Orbit Validation (IOV) satellites, that will be part of the final Galileo configuration, have been launched since 2011, and 22 Full Operational Capability (FOC) satellites are now on order, with the first due to be launched in 2014. The expected operational lifetime of the Galileo satellites will exceed 12 years and the constellation will be completed by the end of this decade. The operational Galileo satellites have revolution periods of about 845 minutes, therefore they complete approximately 17 orbital revolutions during 10 Earth rotations. For this reason they are in a $(17: 10)$ resonance with the Earth's rotation.

\subsection{Beidou}

The Chinese navigation satellite system Beidou (Compass) consists of satellites placed in geostationary orbits, inclined $\left(55^{\circ}\right)$ geosynchronous orbits (IGSO) and near circular 21,528 km medium Earth orbits. The first phase of the program encompassed the creation of a regional system, covering China and neighboring countries, with 5 GEO, 5 IGSO and 4 MEO satellites. The second phase envisages the creation of a global navigation system, with 5 GEO, 3 IGSO and 27 MEO satellites to be deployed by 2020. The MEO satellites (BeidouM) will be placed in 3 orbital planes separated by $120^{\circ}$ at the equator, with 9 satellites in each plane. The selected orbit inclination is $55^{\circ}$, as for GPS. As of 8 July 2013, 5 Beidou-M have been successfully launched in MEO: the first (M1) carried out on-orbit tests, while the following 4 , launched in pairs (M3 and M4, M5 and M6), already belong to the operational model, with a design operational lifetime of 5 years. The Beidou-M satellites have revolution periods of about 773 minutes, therefore they complete approximately 13 orbital revolutions during 7 Earth rotations. For this reason they are in a (13:7) resonance with the Earth's rotation.

\section{Constellation Maintenance}

\section{1. $G P S$}

The design lifetime of the satellites significantly improved over the years going from 5 years for Block I up to 15 years for the recent Block III. The Block IIF spacecraft - 12 in total, of which 3 already in orbit - will remain operational until 2025-2030, while the Block III spacecraft - 32 planned, of which 4 already ordered - should guarantee the navigation services up to around 2040. In all the Block I and II launches, the satellites were deployed into an elliptical Hohmann transfer orbit with low perigee, so they were placed in the final operational nearly circular orbit in MEO through an apogee maneuver carried out with an integrated propulsion system with no release of upper stages around the constellation altitude. The upper stages were removed quite effectively from the MEO region of interest by the effects of the perturbations. The first three Block IIF satellites were instead launched with Delta-4 and the upper stage was used to deploy them in their final orbit. After mission completion, each stage was re-orbited several hundred kilometers above the constellation altitude. This new mission profile will be adopted for the remaining Block IIF satellites and for the Block III satellites as well. In conclusion, since 1978 an average of 7 satellites every 4 years have been placed into orbit. During the last 15 years, characterized only by constellation maintenance, the launch rate slightly decreased to 3 satellites every 2 years. In all cases every launch put into orbit a single satellite. This launch strategy will continue to be implemented in the foreseeable future. For the replacement launch rate, the figures observed during the last 15 years seem appropriate also for the coming 2-3 decades, with 1-2 launches/satellites per year.

\subsection{GLONASS}

All the Uragan satellites, and all the Uragan-M until 2010, were launched in triplets using the Proton rocket and the upper stages that injected the satellites in their final orbit were left in the middle of the constellation. Following a Proton failure in 2010 two Uragan-M spacecraft were launched in 2011 using two Soyuz/Fregat rockets, while another triplet was put into orbit using Proton. A further Soyuz/Fregat launch, during the same year, placed in orbit the first Uragan-K1 spacecraft. In 
these 2011 launches the upper stages were maneuvered to leave the constellation orbit: the mean altitude of the 3 Fregat upper stages was raised a few hundred kilometers above GLONASS, while that of the Briz-M upper stage of the Proton rocket was lowered by several hundred kilometers. This new trend was confirmed by the Soyuz/Fregat launch of the last Uragan-M spacecraft with the upper stage again re-orbited nearly $400 \mathrm{~km}$ above the constellation. Since 1982, an average of 4 satellites per year have been placed into orbit. During the last 15 years, characterized by constellation restoration, the launch rate slightly decreased to 7 satellites every 2 years. In 2013-2015 the launch of 15 GLONASS satellites is planned. Six Uragan-M will be launched by two Proton rockets, while the other six will be put into orbit by an equal number of Soyuz/Fregat. Three launchers of the latter type will also be used to deploy three Uragan-K satellites. However, if the "stabilization" of the operational constellation will be successful, the launch rate is expected to decrease in the future to about 2-3 satellites per year, probably using Soyuz/Fregat single shots or equivalent new launchers.

\subsection{Galileo}

All four launches carried out as of 8 July 2013 used the Soyuz/Fregat combination: the two GIOVE satellites were launched separately, while the following four Galileo-IOV satellites were launched in couples. After having deployed the satellites in their near circular operational orbits in MEO, the Fregat stages were re-orbited to disposal orbits a few hundred kilometers above the constellation altitude and outside the GNSS MEO region. Following their decommissioning, the same disposal strategy was adopted for GIOVE-B and, to a lesser extent, for GIOVE-A. The deployment of the operational constellation will be obtained by launching the 22 Galileo-FOC satellites with 5 Soyuz/Fregat (2 satellites per launch) and 3 Ariane-5ES (4 satellites per launch). The launch rate in this phase will be close to 4 satellites per year. After the completion of the constellation, a replacement rate comparable to that of GPS seems reasonable, i.e. about 2 satellites (1 double launch) per year.

\subsection{Beidou-M}

In the launches carried out in MEO as of 8 July 2013, the satellites were deployed into an elliptical Hohmann transfer orbit, so they were placed in the operational nearly circular orbit in MEO through an apogee maneuver carried out with an integrated propulsion system. The perigee of the CZ-3A upper stage used to deploy Beidou-M1 had a relatively high altitude, so the decay of the apogee was not very fast, even though it is presently about $500 \mathrm{~km}$ lower than the constellation altitude. The mission profile of the following double launches with the CZ-3B rocket was basically the same, but with a much lower transfer orbit perigee height, leading to a quite fast decline of the upper stages apogee. This approach will be probably confirmed in the future. Taking into account the requirement to complete the constellation by 2020 and the relatively short design lifetime of the satellites, 2-3 double CZ-3B launches per year should be expected until the end of the decade, during the deployment phase, and a similar launch rate would be needed to maintain the constellation fully operational in the following years. Only when new spacecraft models with a significantly higher design lifetime will become available, the replacement rate will be free to converge towards the GPS value.

\section{Disposal Practices}

Following the finding that the disposal orbits used by the GNSS satellites in MEO are potentially unstable [2], several studies were carried out to investigate the initial conditions leading to instability, i.e. a significant increase of eccentricity in a few decades, due to resonance conditions from the combined gravitational interaction of geopotential harmonics, Moon and Sun [3] [4] [5] [6]. It was found, for example, that the disposal strategy initially recommended for the GPS satellites was not able, in general, to prevent the long-term crossing of the altitude shells used by the operational spacecraft of other GNSS constellations in MEO, including GPS. Solutions were proposed in order to delay the penetration of the GNSS altitude shells through the minimization of the long-term eccentricity growth [5] [7] [8] [9]. As it strongly depends on the initial elements of the disposal orbit, the solutions discussed focused on the most appropriate choice for the initial eccentricity (very small) and argument of perigee, not too expensive from the point of view of end-of-life propellant consumption. Alternatively, it was also suggested that high eccentricity growth strategies might be adopted, leading to a long-term reduction in the combined constellation and intragraveyard collision risks, compared to the minimum 
eccentricity growth approach [8] [9]. Several recent papers addressed the problem of end-of-life disposal of GNSS spacecraft and the associated collision risk (e.g., [9] [10] [11] [12]).

\section{1. $G P S$}

As of 8 July 2013, a total of 26 GPS satellites had been abandoned in orbit. In addition 3 Delta4 upper stages used to launch the first three new Block-IIF spacecraft were present in MEO. All the previous solid rocket motor upper stages, used with the Atlas-F and Delta-II launchers, had been placed in low perigee elliptical Hohmann transfer orbits, so only 5 of them had an apogee still higher than $17,000 \mathrm{~km}$. Moreover, their number will continue to decrease in the future: two in 2050 and only one in 2150 [12]. Concerning the 26 GPS satellites abandoned in MEO, apart from NAVSTAR 5, which was left in the original operational orbit, and NAVSTAR 2 and 6 , which were maneuvered, respectively, about 90 and $250 \mathrm{~km}$ below, all the other 23 satellites were moved at the end-of-life in higher orbits, between 220 and $1440 \mathrm{~km}$, on average, above the nominal operational altitude of $20182 \mathrm{~km}$. However, no strategy leading to the control of eccentricity growth was implemented, so the abandoned satellites already cross the GLONASS, GPS and Beidou-M altitudes, with an object density peak just below the Beidou-M operational height [12]. The Galileo altitude will be crossed as well in less than 50 years. Concerning the 3 Delta- 4 upper stages, abandoned in MEO since 2010, they were re-orbited between 880 and $1030 \mathrm{~km}$ above the GPS constellation nominal altitude, but again no strategy leading to the control of eccentricity growth was implemented, so the long-term crossing of the Beidou-M and Galileo operational heights will be unavoidable [12].

\subsection{GLONASS}

There were 95 abandoned GLONASS satellites in orbit, as of 8 July 2013. All were left in the operational orbit. In addition, 90 rocket bodies and released tanks associated with the GLONASS launches were still present in MEO with an apogee higher than $17,000 \mathrm{~km}$, including 40 Proton upper stages left in the constellation orbits after spacecraft deployment. Therefore, 135 old satellites and upper stages were not disposed outside the constellation, but just left there at the end-of-life.
Presently the adopted "non-disposal" strategy obviously creates a sharp object density peak at the constellation operational altitude, but no other navigation constellation in MEO is crossed by abandoned GLONASS spacecraft and rocket bodies. This situation will not change for at least another 50 years. The crossing of the GPS altitude will need approximately one century to occur, while only after around 150 years the Beidou-M and Galileo altitudes will be affected as well [12]. As mentioned in Sec. 3, in the last launches the upper stages were raised above the operational orbit. At present it is not clear if the new disposal strategy will become a future standard or not and if it will affect the endof-life disposal of the satellites as well. Probably the requirement to appropriately dispose the Fregat upper stages used to deploy the Galileo satellites played a role in devising the new mission profile. Anyway, even though the mean altitudes of the five upper stages will not change for at least 200 years, the evolution of the eccentricity will be strongly affected by the initial conditions, with a significant long-term impact on the apogee and perigee altitudes [13].

\subsection{Galileo}

For the Galileo satellites and upper stages launched so far, a disposal strategy aiming at constraining the long-term eccentricity growth was implemented, in order to avoid the crossing of the operational altitudes of the navigation constellations in MEO. However, this low eccentricity growth condition was guaranteed for at least 200 years in the case of the GIOVE spacecraft and their upper stages, while for the upper stages used to launch the Galileo-IOV spacecraft the same condition will be met for 100 years. In future Soyuz launches, the injection of the two satellites will occur in a circular orbit $300 \mathrm{~km}$ above the operational constellation and the Fregat upper stage will be abandoned there. In the Ariane-5 launches, the injection of the four satellites will instead occur in a circular orbit 300 $\mathrm{km}$ below the constellation, and the ES (Evolution Storable) upper stage modified for Galileo will be left in such lower orbit. In both cases, it will not be possible to accurately target an optimal argument of perigee, so the goal for the upper stages will be to obtain an orbit as circular and stable as possible, with the injection errors $(3 \sigma)$ of $100 \mathrm{~km}$ in $a$ and 0.0018 in $e$ for the Soyuz/Fregat and $75 \mathrm{~km}$ in $a$ and 0.0012 in $e$ for the Ariane-5ES [14]. 


\subsection{Beidou-M}

It is not clear if the test-bed satellite Beidou-M1 is still functional or not. In any case, it still occupies the constellation nominal orbit. Concerning the two CZ-3B upper stages used to put into orbit the four operational spacecraft, they had been placed in low perigee elliptical Hohmann transfer orbits, so their apogee heights are already more than 800 $\mathrm{km}$ lower than the GLONASS constellation, i.e. the lowest of the navigation systems in MEO, and due to the effects of perturbations, mainly air drag at the perigee, the apogee altitudes will keep decreasing. For the single CZ-3A upper stage used to inject into orbit Beidou-M1, the relative height of the perigee prevented a comparably rapid decay of the apogee, still crossing the GLONASS constellation, but the expected residual lifetime is $\approx 10$ years [11]. In conclusion, all the Beidou-M upper stages have been placed so far in elliptic and short lifetime orbits. Concerning the satellites, however, the endof-life disposal strategy is not yet clear.

\section{Dynamics in the MEO Region}

In the framework of the ESA/GSP Contract, a study of the dynamics in the MEO region was performed in order to obtain a map of the resonances affecting the orbit of the constellations. This shall help in defining the future disposal strategies, either to exploit stable orbits for graveyard or to identify highly unstable orbits for faster re-entry into the atmosphere. For this purpose, a number of orbits representative of the dynamical configurations that can be found in the MEO and GTO regions were propagated and compared with numerical references and TLE times series.

In most of the cases it is possible to establish that the variations of the eccentricity depend upon the initial value of the eccentricity, and mainly of the kind of variation of the perigee (libration or circulation). Some of the variations are related as well to the variations induced on the inclination, since the quantity $\sqrt{1-e^{2}} \cos i$ remains nearly constant. A thorough numerical and analytic analysis of the dynamics related to the coupled variation of these orbital elements, mainly eccentricity and argument of perigee, was performed. For a complete analysis of the problem the reader can see [15]. In Fig. 1, we show a sample result showing the maximal eccentricity reached when changing the initial values of the eccentricity and the argument of perigee for a

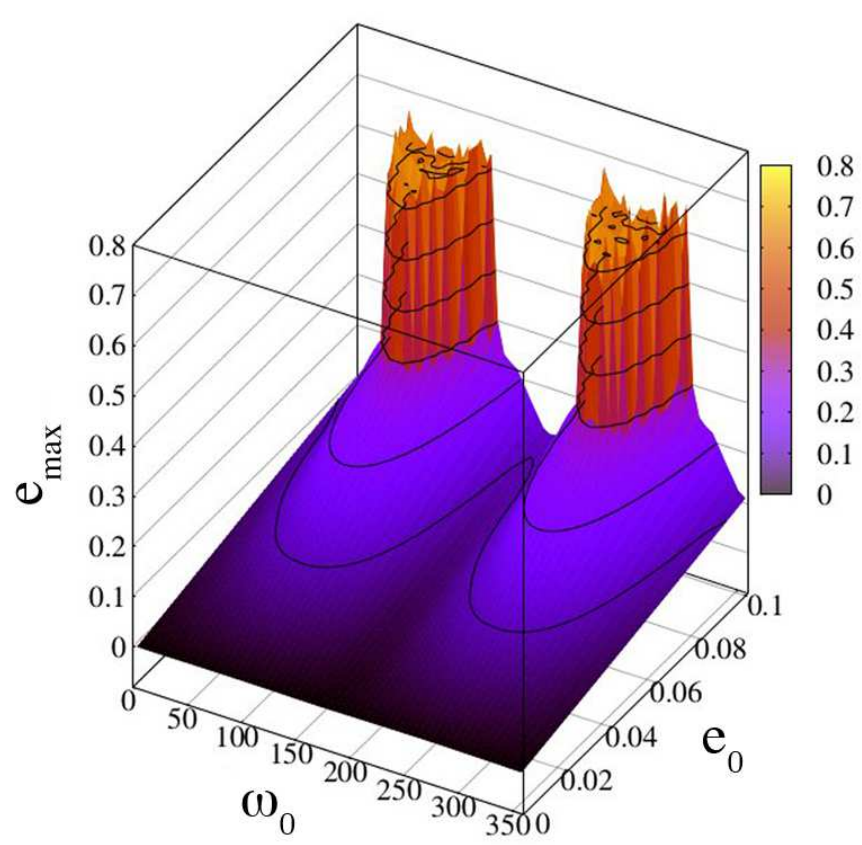

Figure 1: Map of the maximal eccentricity $e_{\max }$ reached when changing the initial values of the eccentricity $e_{0}$ and the argument of perigee $\omega_{0}$ for a GLONASS orbit. The color bar refers to the value of $e_{\max }$.

GLONASS spacecraft. It can be noticed the sensitivity of the dynamics to small changes in the value of the initial $\omega$, leading to a large growth in the final eccentricity in about 200 years for some $\omega$. Clearly this kind of results can be exploited for the optimal choice of the unstable disposal orbits.

\section{De-orbiting with Non-Gravitational Forces}

A number of innovative options in which deorbiting from MEO can be obtained with LowThrust (LT) propulsion or with the exploitation of solar radiation pressure-augmentation devices were studied and will be simulated in the future. Moreover, the coupling of these two strategies was investigated.

\subsection{De-orbiting with Low-Thrust Propulsion}

The preliminary analysis is based on a continuous thrust from a circular orbit in the MEO range to a low Earth orbit with a perigee altitude of $200 \mathrm{~km}$. The simulation model is based on combination of averaging with an analytical solution of low-thrust 
motion in non-singular orbital elements including the effect of solar pressure, $J_{2}$ and the eclipse periods encountered during de-orbiting [16]-[19]. A simple control parametrization is introduced, which aims at ensuring sufficient flexibility while keeping the number of degrees of freedom low. This envisions dividing each orbit into 4 sectors: a perigee thrusting arc, an apogee thrusting arc and two coasting arcs in between. The first, of amplitude $\Delta L_{p}$ around the pericenter, is meant ideally to alter apocenter altitude by thrusting in either way along the tangential direction. Similarly, the second alters the pericenter altitude by thrusting tangentially around the apoapsis for an arc of amplitude $\Delta L_{a}$. The amplitude of the $\operatorname{arcs} \Delta L_{p}$ and $\Delta L_{a}$ are the quantities to be set to define a control profile.

In the following simulations, the de-orbiting with LT propulsion of a Galileo-class satellite from MEO is considered. The initial orbit parameters are semi-major axis of $29600 \mathrm{~km}$, zero eccentricity and inclination of $55.3^{\circ}$ with respect to the equator. The target condition to be reached to achieve deorbiting is a pericenter altitude of $200 \mathrm{~km}$. It is assumed that, once this condition is achieved, the residual atmospheric drag will naturally make the orbit decay until the spacecraft re-enters the atmosphere. Initial spacecraft mass, inclusive of the LT propulsion system and its propellant, is assumed to be $1000 \mathrm{~kg}$. The maximum thrust of the propulsion system is not defined a priori, but it is adjusted according to the required de-orbiting time. The engine's specific impulse is assumed to be $3000 \mathrm{~s}$, which is typical of state-of-the-art electric propulsion systems. The thrusting strategy adopted envisions a thrusting arc at apogee with thrust along the negative tangential direction (i.e., decelerating), together with a thrusting arc at perigee with thrust along the positive tangential direction (i.e., accelerating). These have the combined effect of lowering the perigee, while concurrently raising the apogee, thereby increasing the orbit's ellipticity while retaining a net decrease of the semi-major axis. Figure 2 shows the required engine thrust to achieve de-orbiting in a range of times between 2.5 and 25 years, for different amplitude of the apogee/perigee thrusting arcs. On can see that, if the thrust is applied continuously for the full orbit (ochre line), the engine thrust requirement is around $0.05 \mathrm{~N}$ for the minimum time of 2.5 years and is $0.0025 \mathrm{~N}$ for 25 years. The required thrust is within the capabilities of current electric propulsion systems. Note also that the required thrust decreases exponen- tially with the de-orbiting time, and thus shows a linear behaviour if plotted in a logarithmic scale. On the other hand, thrusting for the full orbit is somewhat less efficient in terms of propellant consumption, as shown in Fig. 2, since the required mass, $85 \mathrm{~kg}$, is some $60 \%$ higher than the case in which the thrust is applied just for one sixth of each revolution (blue line), i.e., $53 \mathrm{~kg}$. On the other hand, in this latter case, the required thrust is more than double than the "full orbit" case. In this sense, there is a trade-off between required thrust and propellant consumption.
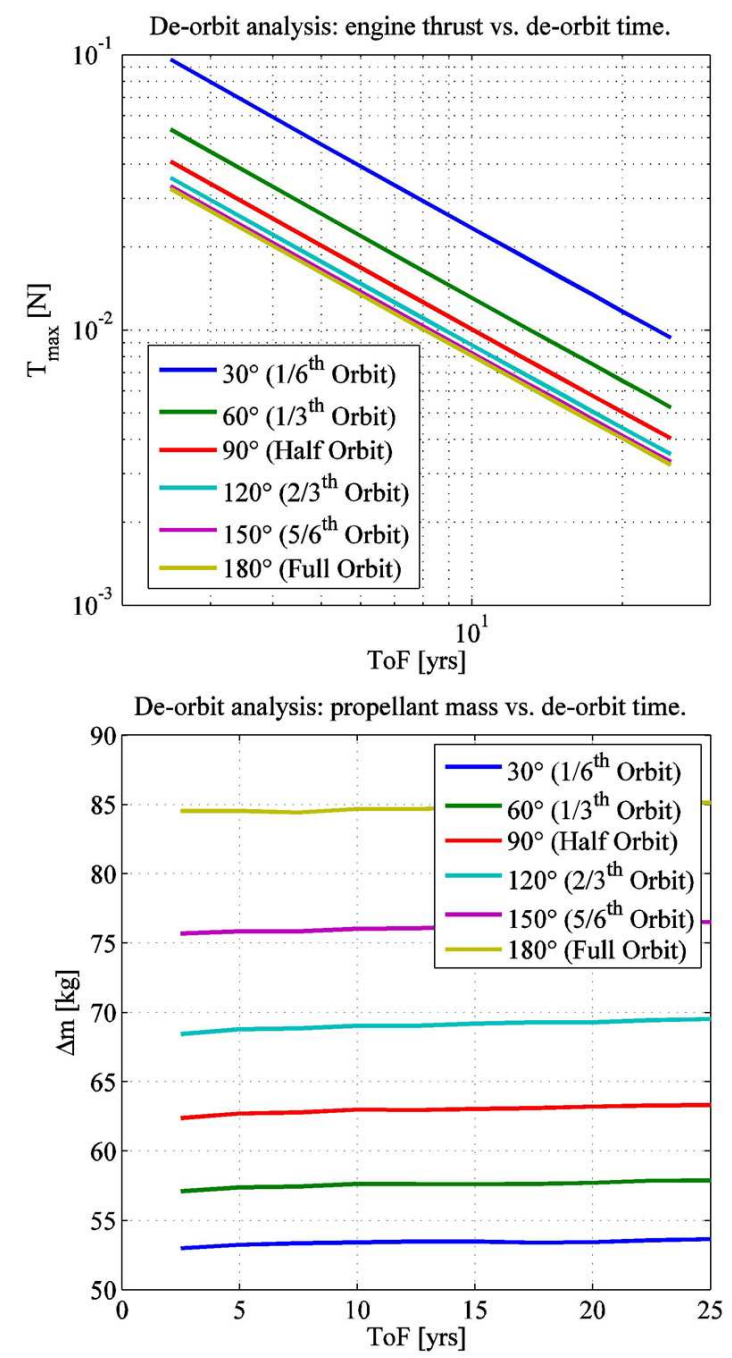

Figure 2: De-orbiting analysis for transfer times between 2.5 and 25 years. a) Required engine thrust in logarithmic scale. b) Propellant consumption. 


\subsection{De-orbiting with Solar Radiation Pressure}

De-orbiting scenarios from MEO exploiting the effect of Solar Radiation Pressure (SRP) were studied too. At MEO altitudes, perturbations such as aerodynamics drag or the electrodynamic drag force are not exploitable since their effect decreases rapidly with increasing distance from the Earth. Past works [20], [21] studied the possibility to exploit the effect of SRP on high altitude orbits to naturally increase the orbit eccentricity, so that, at constant semi-major axis, the orbit perigee can be decreased to cause the spacecraft to re-enter in the Earth atmosphere. Future missions in MEO region could consider increasing the area-to-mass ratio of the spacecraft with some inflatable or deployable device in order to artificially increase the effect of solar radiation pressure for a fast increase of the eccentricity. Note that if an inflatable reflective balloon in employed, it does not require any control after deployment and the de-orbiting (until re-entry in atmosphere) can be completely passive. The orbital dynamics model employed is fully described in [22], for conciseness the equations will not be reported here. The perturbing effect of solar radiation pressure (neglecting eclipses) and the Earth's oblateness are considered. In three dimensions the Sun-perigee angle $\phi$ is defined as $\phi=\Omega+\omega-\left(\lambda_{\text {sun }}-\pi\right)$, where $\Omega$ is the right ascension of the ascending node measured on the equatorial plane, $\omega$ is the argument of perigee and $\lambda_{\text {sun }}$ defines the position of the Sun on the ecliptic with respect to the vernal equinox. If the effective area-to-mass ratio of a conventional spacecraft on a circular orbit can be augmented at the end-of-life above a certain value (say $1 \mathrm{~m}^{2} / \mathrm{kg}$ as a reference), the eccentricity of an initially circular orbit can increase by following the natural orbit evolution. In particular, we can search the minimum effective area-to-mass ratio such that the maximum eccentricity reached along the natural evolution equals the critical eccentricity $e_{\text {crit }}=1-\left(R_{E}+h_{p, \text { drag }}\right) / a$, where $R_{E}$ is the radius of the Earth, $a$ is the semi-major axis of the orbit and $h_{p, \text { drag }}$ is the altitude perigee which defines a successful re-entry. Once the minimum perigee altitude is reached through SRP, de-orbiting is ensured by the presence of aerodynamics drag. Note that the dynamics model used here does not include aerodynamic drag and third body perturbations but a successful de-orbiting was defined as reaching a perigee altitude $h_{p, \text { drag }}$ below $200 \mathrm{~km}$. In a real scenario drag will be dominant below $800 \mathrm{~km}$ and will control the final phase of the de-orbiting [23].

In the simple SRP-augmented de-orbiting described above the effect of solar radiation pressure is exploited by artificially increasing the area-to-mass ratio of the spacecraft on an initially circular orbit. The requirement in effective area-to-mass ratio can be defined by computing the minimum effective area-to-mass ratio which allows the eccentricity to grow up to the critical value. An alternative strategy modulates the effect of solar radiation pressure during de-orbiting as a function of the Sun-perigee angle $\phi$. The effect of solar radiation pressure is exploited only when the secular and long-term evolution of the eccentricity is positive, while the areato-mass ratio increasing device is de-activated otherwise. In this way, a lower area-to-mass ratio is required to reach the critical eccentricity, as more than one cycle in the phase space are allowed. The number of cycles is strictly fixed by the maximum time allowed for de-orbiting and determines also the number of time the area-to-mass ratio increasing device needs to be activated/deactivated. Such an effect can be achieved by changing the attitude of a solar sail with respect to the Sun on an average of 6 months, or by designing a reflective surface with a pyramidal shape, whose area can be controlled. If a simple planar solar sail is chosen, the attitude of the sail must be kept Sun-pointing during the on-arcs, whereas the normal to the sail must be kept perpendicular to the Sun-spacecraft line during the off-arc, so that the effect of SRP is minimized when this would decrease the eccentricity. This effect is also enhanced by the effect of eclipses. Figure 3 shows the requirement in terms of effective area-to-mass ratio for a 5 year de-orbiting with SRP modulation for a Galileo-like spacecraft to de-orbiting until $200 \mathrm{~km}$ (drag is not considered here). A coefficient of reflectivity equal to 1 was considered. SRP-modulation allows decreasing the required area-to-mass ratio and the requirements for Galileo-like spacecraft are as low as 11.5 $\mathrm{m}^{2} / \mathrm{kg}$; also the dependence on the initial condition in terms of $\Omega$ and $\lambda_{\text {sun }}$ is weaker as more cycle in the phase space are covered.

\subsection{De-orbiting with Low-Thrust Propulsion and Solar Radiation Pressure}

The concurrent use of LT propulsion and the SRP was also analyzed. In this scenario, the spacecraft is provided with both an electric engine and an areato-mass ratio augmentation device. The simulation 


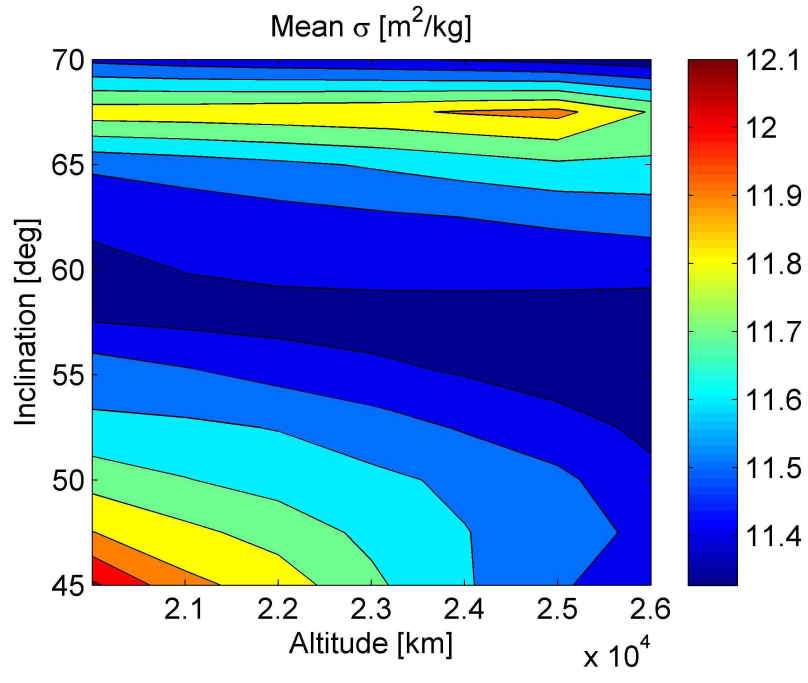

Figure 3: Required effective area-to-mass ratio to de-orbiting with SRP-modulation $[\mathrm{m} 2 / \mathrm{kg}]$. Mean effective area-to-mass ratio.

strategy is therefore similar to that adopted in Sec. 6.1 but this time the contribution of SRP is also added in the propagation. In addition, it is assumed that the SRP modulation strategy described in Sec. 6.2 is adopted. Figure 4 shows the required thrust and mass for a spacecraft with an area-to-mass ratio of $1 \mathrm{~m}^{2} / \mathrm{kg}$. The results are plotted against the LT only case shown in Sec. 6.1.

The results show that the contribution of the SRP consistently helps in reducing thrust and propellant requirements. Moreover, the impact of the SRP contribution increases with the allowed deorbiting time. In this sense, while for 2.5 the gains due to SRP are negligible, for 25 years, the propellant savings reach around $50 \%$ and the thrust is also more than $50 \%$ lower. However, due to operational constraints, it may be convenient to limit the low-thrust action to 6 months; afterwards, an areato-mass ratio increasing device can be deployed to continue the de-orbiting through SRP-modulation only. Figure 5 shows the requirements in terms of area-to-mass ratio and thrust for a de-orbiting from Galileo-like orbit down to $200 \mathrm{~km}$ in a range of 5 years and 6 months to 15 years and 6 months. Two strategies of low-thrust and SRP modulation were investigated:

- 6 months of continuous low-thrust along the anti-tangential direction, in order to decrease the energy and spiraling down with a quasicircular orbit; then SRP modulation (see Fig.
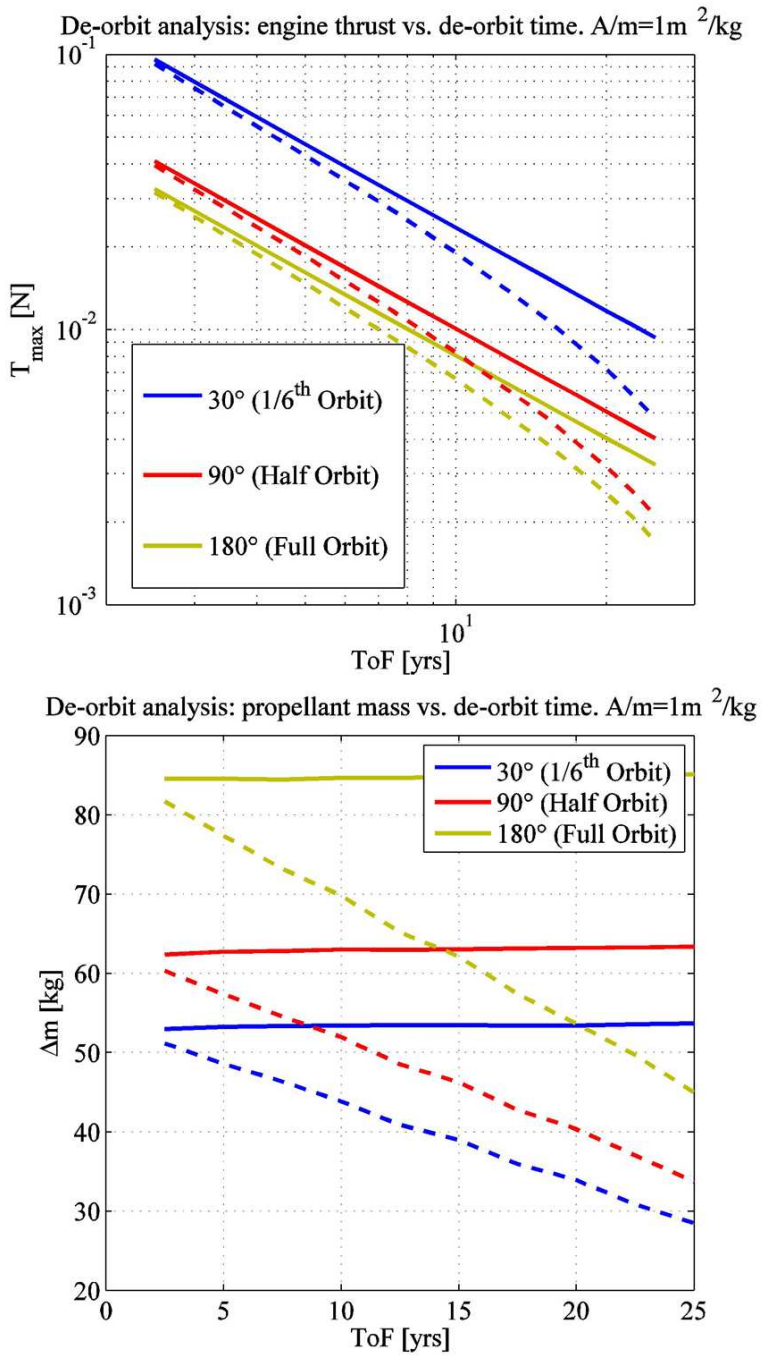

Figure 4: De-orbiting analysis for transfer times between 2.5 and 25 years. a) Required engine thrust in logarithmic scale. B) Propellant consumption. Solid line: LT only. Dashed line: $\mathrm{LT}+\mathrm{SRP}$.

5, panel b).

- 6 months thrusting strategy against the velocity vector across the apogee and along the velocity vector across the perigee to increase the eccentricity; then SRP modulation (see Fig. 5, panel b).

As expected the low-thrust strategy aiming at increasing the eccentricity achieves better results as can be seen by comparing the top and bottom panels of Fig. 5. Effective area-to-mass ratio around $8 \mathrm{~m}^{2} / \mathrm{kg}$ are needed for a de-orbiting in 5 years of SRP modulation, after a 6 -months active de- 

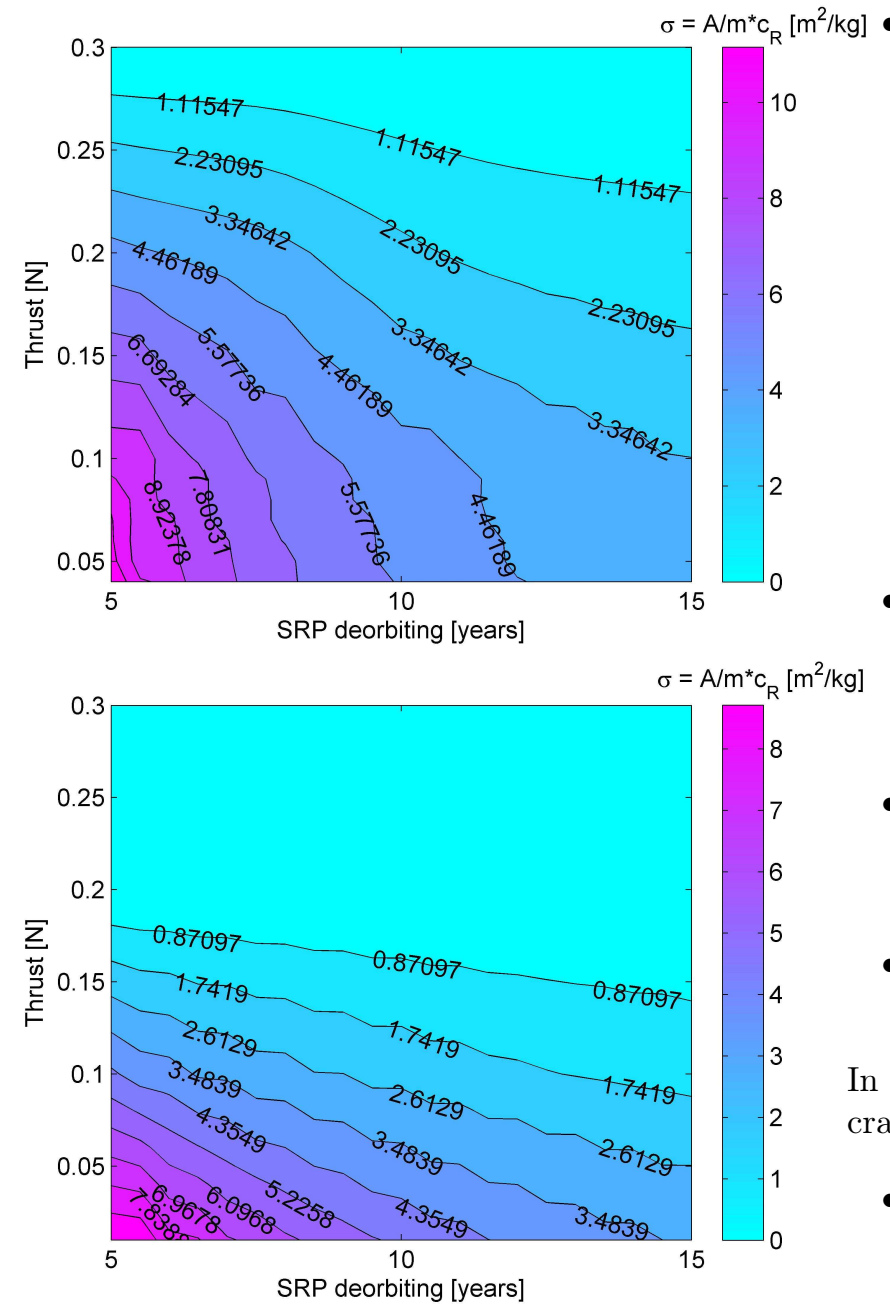

Figure 5: Thrust and effective area-to-mass ratio requirements for coupled low-thrust and SRP modulation deorbiting. a) 6 months of continuous low-thrust along the anti-tangential direction, then SRP modulation. b) 6 months thrusting strategy against the velocity vector across the apogee and along the velocity vector across the perigee, then SRP modulation.

orbiting with $0.03 \mathrm{~N}$ low-thrust. If the thrust level is increased and the time constraints on the deorbiting phase with SRP are relaxed, effective areato-mass ratio values around $3-1 \mathrm{~m}^{2} / \mathrm{kg}$ are enough. Note, again, that in this simulation the effect of drag on the de-orbiting was not considered so the actual requirement in terms of area-to-mass ratio are lower; this choice was made to ensure a conservative design to other perturbations neglected and to solar activity and eclipses.

In conclusion, opportunities for de-orbiting circular MEO spacecraft include:
- SRP augmented de-orbiting (passive). This passive de-orbiting strategy has been shown to be feasible for small spacecraft. Due to the exploitation of SRP and drag, this strategy is characterized by uncertainty in the reentry point. For this reason it can be applied to low-mass and low-re-entry survival chances. It should also be considered that an increased area-to-mass ratio increases the risk of collision during the de-orbiting (higher for drag augmentation), however it shortens the deorbiting time and in some cases it makes reentry achievable.

- SRP augmented de-orbiting (active). SRP modulation allows decreasing the area-to-mass ratio requirements but needs a system solution for multiple deployments.

- Low-thrust can provide de-orbiting with reasonable thrust level and mass consumption if the de-orbiting time is long enough.

- Low-thrust + SRP combination allows savings in engine size and propellant (up to 40-50\%).

In the case of de-orbiting of a Galileo-like spacecraft:

- SRP-modulation would require $11-11.5 \mathrm{~m}^{2} / \mathrm{kg}$ $(100 \times 100 \mathrm{~m}$ for $1000 \mathrm{~kg})$ for 5 years. This strategy would require 5 to 6 cycles of folding/deploying an area-to-mass ratio increasing device every $\sim 6$ months. Allowing a longer deorbiting time would decrease the area-to-mass ratio requirements to $2-2.6 \mathrm{~m}^{2} / \mathrm{kg}(44 \times 100 \mathrm{~m}$ for $1000 \mathrm{~kg}$ ) for 25 years

- Low thrust can de-orbit in 5 years with as little as $16-47 \mathrm{mN}$ thrust and $53-85 \mathrm{~kg}$ of propellant; in 25 years with as little as $3.2-9.3 \mathrm{mN}$ thrust and $53-85 \mathrm{~kg}$ of propellant.

- Low thrust+SRP modulation can de-orbiting in 5 years with as little as $15-43 \mathrm{mN}$ thrust and $77-48 \mathrm{~kg}$ of propellant with $1 \mathrm{~m}^{2} / \mathrm{kg}$ areato-mass ratio; in 25 years with as little as $1.7-$ $4.7 \mathrm{mN}$ thrust and $28-45 \mathrm{~kg}$ of propellant with $1 \mathrm{~m} 2 / \mathrm{kg}$ area-to-mass ratio.

- 6 months low thrust + SRP modulation can deorbit in 6 years and 6 months with $0.1 \mathrm{~N}$ thrust and $3.83 \mathrm{~m} 2 / \mathrm{kg}$ of effective area-to-mass ratio 
increase. For a $1000 \mathrm{~kg}$ spacecraft, this corresponds to a $61.9 \mathrm{~m}^{2}$ sail if the reflectivity coefficient is equal to 1 , but decreases to $46.15 \mathrm{~m}^{2}$ for 1.8 reflectivity coefficient. The de-orbiting time can be lower, for example 5 years and 6 months with lower thrust level is $0.08 \mathrm{~N}$ and $5.60 \mathrm{~m}^{2} / \mathrm{kg}$ of area-to-mass ratio device. This corresponds to a $74.85 \mathrm{~m}^{2}$ sail if the reflectivity coefficient is equal to 1 , and $55.79 \mathrm{~m}^{2}$ for 1.8 reflectivity coefficient on a $1000 \mathrm{~kg}$ spacecraft. Other options may be selected in Fig. 5.

Future work for this analysis can extend to combine the effect of solar radiation pressure with resonances of the gravity field; i.e., area-to-mass ratio increasing devices can be used to passively achieve a target inclination/eccentricity where resonances of the full Earth gravity field and third body perturbations can be exploited for the final stages of de-orbiting. Moreover, the present study will be extended to MEO elliptical orbits. An analysis of the increase in collision risk due to the augmented spacecraft cross section will be done as well.

The SRP can be also exploited to reach graveyard stable orbits, in this case smaller area-to-mass ratio would be required.

\section{Maneuver Rate Evaluation}

One of the final goals of the study will be the evaluation of the frequency of the maneuvers required to avoid collisions or close approaches with space debris and old spacecraft in the GNSS region. An approximate preliminary estimation of this value can be obtained by analyzing the collision risk and the orbital crossing as obtained by the CUBE algorithm [24]. On the other hand a more refined algorithm, based on [25] and [26] was developed and implemented. A brief description of the algorithm follows.

\subsection{Probability of Collision}

Let us consider two nominal objects of radius $R_{1}$ and $R_{2}$, respectively, and let us assume that at time $t=t_{c a}$ they experience a close approach, being $\mathbf{r}_{c a}$ and $\mathbf{v}_{c a}$ the corresponding relative position and velocity. Let us also assume that the variables in the phase space associated with the two bodies follow a Gaussian distribution. The joint probability density function in position in the neighborhood of the close approach can be thus be defined as [26]

$$
p(\mathbf{r})=\frac{\sqrt{\operatorname{det}(C)}}{2 \pi^{3 / 2}} \exp \left(-\frac{1}{2}\left(\mathbf{r}-\mathbf{r}_{c a}\right)^{t} C\left(\mathbf{r}-\mathbf{r}_{c a}\right)\right)
$$

where $C$ is the normal matrix corresponding to $\mathbf{r}_{c a}$. The probability of collision is then the volume integral of $p(\mathbf{r})$, namely

$$
P=\int_{V} p(\mathbf{r}) d x d y d z
$$

where $V=4 / 3 \pi R_{c}^{3}$ with $R_{c}=R_{1}+R_{2}$.

By introducing the concept of target plane, this probability can be reduced to a two-dimensional integral [25]. We recall that the target plane is the plane containing one of the two bodies, say the target, and perpendicular to the relative velocity vector [26]. A collision can be seen as an orbit containing a point in the target plane inside the circular cross section $A_{c}=\pi R_{c}^{2}$.

Moreover, from orbit determination theory we know that the confidence ellipsoid centered at a given solution $\mathbf{x}^{*} \in \mathbb{R}^{n}$ is defined as

$$
\left\{\mathbf{x} \in \mathbb{R}^{n}:\left(\mathbf{x}-\mathbf{x}^{*}\right)^{t} C\left(\mathbf{x}-\mathbf{x}^{*}\right) \leq \sigma^{2}\right\},
$$

where $\sigma>0$ is a given parameter. In other words, the boundaries of the confidence ellipsoid are the level surfaces of the probability density function.

In our simulations we consider that a well-defined covariance matrix $\Gamma\left(t_{0}\right)=C^{-1}\left(t_{0}\right)$ is associated with each body at some initial time $t=t_{0}$ in some reference system. At $t_{c a}$, we have

$$
\Gamma\left(t_{c a}\right)=\Phi\left(t_{0}, t_{c a}\right) \Gamma\left(t_{0}\right) \Phi\left(t_{0}, t_{c a}\right)^{t},
$$

where $\Phi\left(t_{0}, t_{c a}\right)$ is the state transition matrix associated with the nominal object, obtained by propagating the equations of motion together with the corresponding variational equations from $t_{0}$ to $t_{c a}$. In our software we implemented the option to choose among different dynamical systems, namely the two body problem, the two body problem plus either the effect due to $J_{2}$ or the atmospheric drag or both of them. An exponential atmospheric density model is considered.

To compute $C$ needed by (1), the confidence ellipsoids corresponding to the uncertainty in position for the two bodies are propagated up to the same time and then $\Gamma\left(t_{c a}\right)=\Gamma_{1}\left(t_{c a}\right)+\Gamma_{2}\left(t_{c a}\right)$ represents covariance matrix accounting for the position uncertainty of both objects. 
To compute the probability of collision as an area integral, the axes of the target plane are defined [27] as

$$
\hat{\mathbf{x}}_{t p}=\frac{\mathbf{r}_{c a}}{\left\|\mathbf{r}_{c a}\right\|}, \quad \hat{\mathbf{y}}_{t p}=\frac{\mathbf{r}_{c a} \times \mathbf{v}_{c a}}{\left\|\mathbf{r}_{c a} \times \mathbf{v}_{c a}\right\|}
$$

The relative position of the two objects at $t_{c a}$, the common covariance matrix and its inverse are projected onto this plane obtaining $\mathbf{r}_{t p}^{*}, \Gamma_{t p}$ and $C_{t p}$, respectively. Then, the probability of collision over the cross section of radius $R_{c}$ centered at the predicted closest approach $\mathbf{r}_{t p}^{*}$ is

$P=\frac{1}{2 \pi \sqrt{\operatorname{det}\left(\Gamma_{t p}\right)}} \int_{-R_{c}}^{R_{c}} \int_{-\sqrt{R_{c}^{2}-x^{2}}}^{\sqrt{R_{c}^{2}-x^{2}}} \exp \left(-\frac{1}{2} \Delta \mathbf{r}^{t} C_{t p} \Delta \mathbf{r}\right) d y d x$

where $\Delta \mathbf{r}=\mathbf{r}_{t p}-\mathbf{r}_{t p}^{*}$, being $\mathbf{r}_{t p}$ another vector in the target plane.

\subsection{Maneuver Rate}

Once we know the probability of collision for a couple of objects we compare the value with a threshold given in input. If the threshold is exceeded we assume that a maneuver must take place. In this way we get the maneuver rate for the investigated time span. Comparing the number of collisions obtained by the CUBE algorithm with the events for which the maneuver was dictated by the algorithm described above, we obtain the number of avoided collisions. The cases were a collision takes place without the collision avoidance algorithm dictating the maneuver represent the residual collision rate. The false alarm rate can be computed from the above quantities either following Foster's approach or by taking the differences between the performed maneuvers and the actual collisions.

\subsection{Simulation Procedure}

The method described in Sec. 7 was implemented in a post-processing tool, called posrisk, of our long term evolution model SDM [28]. A sketch of the simulation procedure follows. In the final simulation setup the debris population will be a subset of MASTER 2009, containing objects larger than $5 \mathrm{~cm}$. Every 5 days a snapshot of the debris population is taken and a check whether two objects belong to the same $10 \times 10 \times 10 \mathrm{~km}^{3}$ cube first, and within an enlarged cube of $30 \times 30 \times 30 \mathrm{~km}^{3}$ in a second step, is made (both the time step and the size of the cube will be optimized in the course of the simulation campaign). Whenever this happens the collision probability between the two objects is computed with the standard CUBE algorithm. Moreover, in this case we compute if such objects have other close approaches at a relative distance smaller than a user defined distance threshold over an interval $\left[t_{0}-\Delta t: t_{0}+\Delta t\right]$ days (with $\Delta t=1$ day as a default value), being $t_{0}$ the time of the snapshot. Whenever this happens, we compute the corresponding probability of collision, within a collision radius $R_{c}$ given by the sum of the projectile and target radii. With respect to $\Gamma\left(t_{0}\right)$, we have considered a diagonal matrix, whose entries are the square of the uncertainty in position and velocity in the radial, transversal, out-of- plane $(\mathbf{r}, \mathbf{t}, \mathbf{w})$ reference system. These values shall be given in input by the user according to the SSA system performances. The propagation of the orbits is done in the equatorial reference system, so at each snapshot each $\Gamma_{r t w}\left(t_{0}\right)$ is projected first onto this plane.

\section{Long-term Simulations}

The core of the study will be a set of simulations of the MEO debris environment evolution, performed either with SDM 4.2 or with DAMAGE, two well known long-term evolution codes. The simulations will cover different scenarios that can schematically summarized as follows:

- Business As Usual, reference scenario: the current maintenance and disposal strategies highlighted in Sec. 2 will be implemented and applied.

- Stable orbit targeting: disposal orbits with properly selected $\omega$ will be targeted to minimize the long-term growth of the eccentricity.

- Dilution of collision probability: elliptic disposal orbits, applying different de-orbiting maneuvers (with growing $\Delta V \mathrm{~s}$ ) will be explored, aiming at possibly unstable orbits with the purpose of reaching atmospheric re-entry.

- Exploitation of non-gravitational perturbations: following the results of Sec. 6, a few disposal scenario involving drag augmentation devices will be explored. An analysis of the associated collision risk will be performed too.

- Collision consequences: a scenario where a fragmentation takes place in the MEO region 
will be explored to highlight the possible longterm consequences of this event and the corresponding increase in maneuver rate.

In order to simulate the above scenarios the SDM and DAMAGE software are being adapted to properly reproduce the complex maintenance and disposal practices described in the paper. As an example, the capability to control the RAAN of the constellation planes within a pre-defined window, a refined specific traffic model for constellations building, the possibility to de-orbit the satellites and upper stages in selected disposal orbits are some of the changes introduced up to now.

Simulation campaigns are ongoing mainly to test the new software. As an example, Fig. 6 shows the expected number of collisions (cumulative collision probability) in the altitude band occupied by the GNSS constellation, averaged over 25 Monte Carlo runs of SDM, for three scenarios related to the first 3 cases described in the bullets above. To highlight the effect of the disposal strategies, only the collision risk associated with objects resident in $\mathrm{MEO}$ is shown here, that is objects with perigee in LEO are not considered in this plot. The small number of MC runs is responsible for the large jumps in the curves. Anyhow the reduced collision risk associated with the dilution of collision case is noticeable. Also the de-orbiting in stable, low eccentricity growth, orbits appears favorable in lowering the overall collision risk in the region. Better statistics in terms of MC runs and more refined scenarios are currently under study.

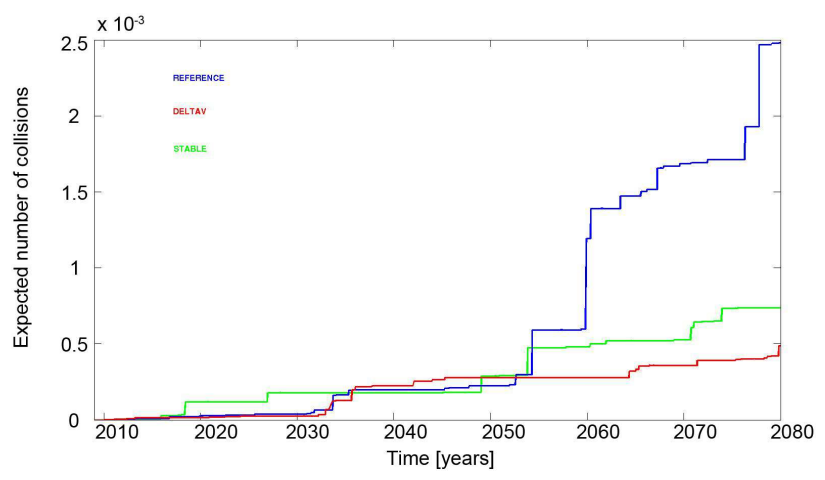

Figure 6: Time evolution of the cumulative collision probability in the altitude band of the GNSS, for three different scenarios described in the text

\section{Conclusions and Future Work}

A comprehensive study of the disposal strategies to be adopted for the GNSS constellation is undergoing under an ESA/GSP Contract. The main focus is on the forthcoming Galileo, but as it is been stressed several times the whole MEO regime must be taken into consideration.

An analysis of the historical practices of the GNSS constellations was performed in order to properly define the reference scenario for the future simulations. The dynamics in the MEO region is under study to define a map of the resonances in the region with the aim of finding the best disposal orbits, either stable or unstable. A number of software upgrades were performed and are undergoing in SDM and DAMAGE to properly simulate the MEO regime and the constellations maintenance and disposal strategies. Moreover, to properly analyze the simulation results in terms of orbital crossing and maneuver rate a new part was added to SDM implementing the Foster's approach [25] to compute the collision risk between two objects taking into account the full, time evolving, covariance matrix.

The final simulation scenarios are currently under definition and the full scale simulations will start soon after the final software review and acceptance. The long-term evolution results will form the basis for the collision risk analysis to be performed with the software described in Sec. 7.

[1] G. Beutler, Methods of Celestial Mechanics, Volume II: Application to Planetary System, Geodynamics and Satellite Geodesy, Springer, Berlin, Germany, 2005.

[2] C. C. Chao, MEO disposal orbit stability and direct reentry strategy, in: Spaceflight Mechanics 2000, Advances in the Astronautical Sciences Series, Vol. 105, Part II, Univelt Inc., San Diego, California, pp. 817-838, 2000.

[3] R. A. Gick, C. C. Chao, GPS disposal orbit stability and sensitivity study, in: Spaceflight Mechanics 2001, Advances in the Astronautical Sciences Series, Vol. 108, Part II, Univelt Inc., San Diego, California, pp. 2005-2018, 2001.

[4] C. C. Chao, R. A. Gick, Long-term evolution of navigation satellite orbits: GPS/GLONASS/GALI-LEO, Adv. Space Res., 34 (2004) 1221-1226.

[5] A. Rossi, Resonant dynamics of Medium Earth Orbits: space debris issues, Celest. Mech. Dyn. Astr., 100 (2008) 267-286.

[6] D. M. Sanchez, T. Yokoyama, P. I. de Oliveira Brasil, R. R. Cordeiro, Some initial conditions for disposed satellites of the systems GPS and Galileo constellations, Math. Probl. Eng., Article ID 510759 (2009).

[7] C. J. Saunders, C. E. Martin, H. G. Lewis, Disposal orbit characteristics for Galileo including orbit propagation techniques, Proceedings of the Fourth European Confer- 
ence on Space Debris, ESA SP-587, ESA Publications Division, Noordwijk, The Netherlands, 2005.

[8] A. B. Jenkin, R. A. Gick, Dilution of disposal orbit collision risk for the medium Earth orbit constellations, Aerospace Report No. TR-2005 (8506)-2, The Aerospace Corporation, El Segundo, California, May 13, 2005.

[9] A. Rossi, L. Anselmo, C. Pardini, R. Jehn, Effectiveness of the de-orbiting practices in the MEO Region, Proceedings of the Fifth European Conference on Space Debris, ESA SP-672, CD-ROM, ESA Communication Production Office, Noordwijk, The Netherlands, 2009.

[10] A. B. Jenkin, R. A. Gick, Collision risk posed to the global positioning system by disposed upper stages, J. Spacecraft Rockets, 43 (2006) 1412-1418.

[11] L. Anselmo, C. Pardini, Orbital evolution of the first upper stages used for the new European and Chinese navigation satellite systems, Acta Astron., 68 (2011) 20662079

[12] C. Pardini, L. Anselmo, Post-disposal orbital evolution of satellites and upper stages used by the GPS and GLONASS navigation constellations: The Long-term impact on the medium Earth orbit environment, Acta Astron., 77 (2012) 109-117.

[13] L. Anselmo, C. Pardini, Review of medium Earth orbit GNSS constellation configuration, operation, maintenance and disposal, Study Note of Work Package 110 Version 1.2, ESA Contract No. 4000107201/12/F/MOS, Disposal Strategies Analysis for MEO Orbits, ISTI/CNR, Pisa, Italy, July 8, 2013.

[14] D. A. Handschuh, B. Carpenter, D. De Chambure, N. Lindon, Stability of the Ariane-5ES Galileo Disposal Orbit, Proceedings of the Sixth European Conference on Space Debris, ESA SP-723, CD-ROM, ESA Communication Production Office, Noordwijk, The Netherlands, 2013.

[15] F. Deleflie, A. Bourgoin, E. M. Alessi, J. Daquin, A. Vienne, V. Morand, D. Hautesserres, A. Rossi, M. Fouchard, Exploration on the Structure of the web of resonance within the MEO and GTO regions, paper AAS 13-788, AAS/AIAA Astrodynamics Specialist Conference, Hilton Head, USA, August 11-15, 2013.

[16] F. Zuiani, Y. Kawakatsu, M. Vasile, Multi-Objective Optimisation of Many-Revolution, Low-Thrust Orbit Raising for Destiny Mission, paper AAS 13-264, 23rd AAS/AIAA Space Flight Mechanics Conference, 2013.

[17] F. Zuiani, M. Vasile, Extension of Finite Perturbative Elements for Multi-Revolution Low-Thrust Transfer Optimisation, paper IAC-12-C1.4.6, 63rd International Astronautical Congress, Naples, Italy, September 2012.

[18] F. Zuiani, M. Vasile, Preliminary design of Debris removal missions by means of simplified models for LowThrust, many-revolutions, transfers. Int. J. Aerospace Eng., Article ID 836250 (2012).

[19] F. Zuiani, M. Vasile, A. Palmas, G. Avanzini, Direct transcription of low-thrust trajectories with finite trajectory elements, Acta Astron., 72 (2012) 108-120.

[20] C. Lücking, C. Colombo, C. R. McInnes, A passive satellite de-orbitinging strategy for Medium Earth Orbit using solar radiation pressure and the $J_{2}$ effect, Acta Astron., 77 (2012) 197-206.

21] C. Lücking, C. Colombo, C. R. McInnes, Solar Radiation Pressure-Augmented Deorbiting: Passive End-of-Life Disposal from High-Altitude Orbits, J. Spacecraft Rockets, doi: 10.2514/1.A32478, in press, 2013.

[22] C. Colombo, C. Lücking, C. R. McInnes, Orbital dy- namics of high area-to-mass ratio spacecraft with $J_{2}$ and solar radiation pressure for novel Earth observation and communication services, Acta Astron., 81 (2012) 137-150.

[23] C. Colombo, C. R. McInnes, Orbital dynamics of 'smart dust' devices with solar radiation pressure and drag, J. Guid. Control Dynam., 34 (2011) 1613-1631.

[24] J. C., Liou, Collision Activities in the Future Orbital Debris Environment, Adv. Space Res., 38 (2006) 21022106.

[25] J. Foster, The Analytic Basis for Debris Avoidance Operations for the International Space Station, Proceeding of the Third European Conference on Space Debris, ESA SP-473, 441-445, 2001.

[26] A. Milani, G.F. Gronchi, Theory of Orbit Determination, Cambridge University Press, 2010.

[27] H. Klinkrad, J. Alarcón, N. Sánchez, Operational Collision Avoidance with Regard to Catalog Objects, in Space Debris: Model and Risk Analysis, Springer, Berlin, Germany, pp. 215-240, 2006.

[28] A. Rossi, L. Anselmo, C. Pardini, R. Jehn, The new Space Debris Mitigation (SDM 4.0) long term evolution code, Proceedings of the Fifth European Conference on Space Debris, ESA SP-672, CD-ROM, ESA Communication Production Office, Noordwijk, The Netherlands, 2009. 